\title{
A Study of Sound Aesthetics in Screenplays of Asghar Farhadi Based on Michel Chion's Theories
}

\author{
Hossein Mashali \\ Islamic Azad University, Iran \\ Leila Montazeri \\ Islamic Azad University, Iran
}

\begin{abstract}
The atmosphere created in a screenplay, how the screenwriter presents it, and how the reader perceives it are some of the most fundamental topics in studying the aesthetics of screenwriting, a significant part of which is writing audio descriptions. This paper discusses one of the most critical and necessary foundations of screenwriting - creating the auditory atmosphere of the film - in eight notable screenplays of Farhadi's films based on Michel Chion's theory with an emphasis on the elements of voice over, acousmatic, acousmetre, music and sound effects, and ambience. This theory deals with materialised signs of sound, which are the details that make us feel the tangible presence of the sound and also know what kind they are, and what the surplus-value is that the auditory atmosphere could never have had without being written down.
\end{abstract}

Keywords: Michel Chion, Screenplay sound design, Sound study, Auditory atmosphere, Writing sounds

\section{Introduction}

One of the main principles of aesthetics in the study of dramatic literature, especially screenplays, is the atmosphere and methods with which it is created. The art and mastery of a screenwriter lie in building an understandable atmosphere, while the difference between screenwriters is undoubtedly in how they use and present expressive tools in writing a script. The written material is divided into the visible atmosphere and invisible atmosphere (outside the frame). The screenplay is an independent part and should be composed in details and with specific writing devices. As much as the script needs to be visualised in writing, the auditory atmosphere should also be tangible and understandable. In addition to dealing with all the other necessary steps, the screenwriter, as the creator of the dramatic work, examines the auditory atmosphere separately and writes down its dramatic functions. That is why classical cinema was limited to the space inside the frame, showing the viewer only the realm of the image inside the screen. However, leading screenwriters and filmmakers sought to deconstruct established principles to create a new perspective in the contemporary era. They have exceeded the visible boundaries in favour of testing and developing new perspectives. Furthermore, they have consciously used these atmospheres in their works according to the invisible spaces. This research will study the details in writing the auditory atmosphere in Asghar Farhadi's selected works based on Michel Chion's theories.

\section{Biography of Asghar Farhadi}

Asghar Farhadi is an Iranian screenwriter and filmmaker who has received two Academy Awards for Best Foreign Language Film for A Separation and Salesman. Farhadi has a realistic style in filmmaking. In his screenplays, he carefully arranges everything to leads us to discover the truth to the degree that he does not even overlook minor elements.

\section{Biography of Michel Chion}

Born in 1947 in Creil, France, he is an experimental composer currently holding the post of an Associate Professor at the University of Paris III, Sorbonne Nouvelle, where he is a theoretician and teacher of audio-visual relationships. He has also written several books on film sound and the interaction between sound and image, which critics considered in 1990 to be the definitive book on the relationship between sound and image.

\section{The dilemma of writing sound in the script}

Writing sounds in a script helps to improve the comprehensible atmosphere. Since it is crucial to write all the aesthetic details in a screenplay to visualise it properly, it will be vital to pay attention to the auditory details to make the visualised text hearable. Sound placement and writing them precisely will help tremendously in creating this atmosphere. Since books and articles have dealt more with sound after the script is written, not many sources are available. But what is clear is that many writers and filmmakers have consciously recorded and written sound in its various natures.

\section{The Atmosphere and Ambient Sounds}

Ambient sound surrounds the scene and occupies the space, like the sound of a forest or a street. It can also be called a regional sound because its inclusive and constant presence helps identify a specific environment and region. In addition to increasing realism, they can also be used to influence emotions.

\section{On-Screen Sounds, Off-Screen Sounds}

In a limited sense and concerning what is displayed in a shot, an off-screen sound is an acousmatic sound, e.g. a sound whose source is - wholly or temporarily - invisible. On the other hand, the source of an on-screen sound is visible and belongs to the 
reality in which it is represented. Michel Chion uses the term non-diegetic sound to identify its tentative source, which is absent in the shot and contradicts the fictional world. A typical non-digestive example is an off-screen dialogue and narration and, of course, musical emphasis (Chion 2017: 42).

\section{Acousmetre}

An acousmetre is an acousmatic character with a certain ambiguous and unstable relationship to the image. We can define it as something that is neither inside nor outside the frame. It is not in the image because the source of sound - the body, the mouth is not in the frame (Chion, 2017: 80). It is not out of the frame either because it does not take an imaginary and hypothetical side, like a moderator or a witness. It is somewhat implicit in the action on the verge of becoming part of the action any minute. It, therefore, can immediately be robbed of its mysterious powers (all-seeing, omniscience, absolute power of influence, omnipresence). At this point, simultaneousness makes the sound realise that it has been limited to a specific body, or in technical terms, has been de-acousmatized. De-acousmatization has a revealing process that is undoubtedly dramatic. (Chion 2017: 30)

\section{Diegetic Sounds}

The sounds produced electronically on the scene are called diegetic sounds - sounds that play on the radio, telephone, tape recorder, etc.

\section{Dancing in the Dust}

Short synopsis: Nazar, in his early twenties, meets his future wife, Reyhaneh, in a minibus ride. They get married, but because there are rumours about Reyhaneh's mother, he is forced to divorce Reyhaneh. To escape from creditors who are after him, he has to share a journey with the old snake hunter. The old man does not want him there at first, but after a poisonous snake bites the young man, he cuts off the snake-bitten finger, takes Nazar to the hospital, and sells his car to pay for the operation.

The radio is playing the Midday Azan. (Farhadi $2014,15)$ \{Diegetic sound\}

The shot begins with a cassette player that is also a disco light. Nazar's finger enters the frame and presses the play button. Song: The Year 2000 by Dariush Eghbali. (Farhadi 2014, 23) \{Diegetic sound\}

The sound of a car comes from outside. It stops behind the stalls and lights insides. \{Off-screen sound\}

From the beginning of the sequence, we hear the guide's explanations on the shots. (Farhadi 2014, 28) \{Off-screen sound\}

O.S.: Are you dead? Nazar turns his head toward the outside. (Farhadi, 2014: 30). \{Off-screen sound\}

The kiosk owner: l'll make it a portable kiosk if you give it to me, and then l'll use it to drive home at night. (Farhadi 2014, 37) \{Off-screen sound\}
The dark desert and animal howling terrify him. (Farhadi 2014, 40) \{Off-screen sound\}

He looks at the old man's car. The faint sound of an old song comes from inside. (Farhadi 2014, 40) \{Diegetic sound\}

Nazar: I swear, the cold weather is killing me - the sound of the song gets louder. (Farhadi 2014, 41) \{Diegetic sound, ambient sound\}

The old man throws a matchbox out and closes the door. The music dies down. (Farhadi, 2014: 41). \{Diegetic sound\}

Nazar is twisting a new thread around the ring. The sound of the Volkswagen's sliding door and the continuous hurling of the old man get Nazar out of his head. He gets up, confused. (Farhadi 2014, 42) \{Off-screen sound\}

The sound of the same old song comes from inside the Volkswagen. (Farhadi 2014, 41) \{Diegetic sound\}

The old man is still calmly blowing the opium smoke towards the snake. The snake came out of the hole but suddenly escaped back when Nazar runs down to this side of the hill shouting and calling the old man. (Farhadi 2014, 44) \{Off-screen sound\}

The old man is pouring oil into the frying pan, pretending he does not hear Nazar. The sizzling sound of oil in the frying pan. (Farhadi 2014, 46) \{Off-screen sound\}

The old man puts on a song, switches the engine, and then lies on a mattress at the back of the car. (Farhadi 2014, 53) \{Diegetic sound\}

The sound of a fire comes from outside. (Farhadi 2014, 53) \{Off-screen sound\}

Through the broken windows, the wind is storming in with a howling sound. (Farhadi 2014, 55) \{Ambient sound\}

The wind stops. He turns on the cassette player. (Farhadi 2014, 55) \{Diegetic sound\}

The sound of the old man's song comes along with the sound of the fierce wind. (Farhadi 2014, 55) \{Diegetic sound, ambient sound\}

He walks a few steps in the dark but suddenly stops when he hears the sound of opening the car's sliding door. (Farhadi 2014, 56) \{Off-screen sound\}

The sound of the song breaks the silence of the desert. (Farhadi 2014, 56). \{Diegetic sound\}

He finds another tape and puts it in the player. Now a cheerful, romantic song is playing. (Farhadi 2014, 56) \{Diegetic sound\}

The desert is in peace. (Farhadi 1393, 57) \{Ambient sound\}

The snake escapes back into the hole when Nazar claps joyfully. (Farhadi 2014, 57) \{Off-screen sound\}

Nazar's voice comes from outside the car. (Farhadi 2014, 59) \{Off-screen sound\}

The old man gets out of the car and listens closely to hear Nazar. (Farhadi 2014, 61) \{Off-screen sound\}

A shot of the old man's car while the sound of the song comes from it. (Farhadi 2014, 62) \{Diegetic sound, ambient sound\}

The music still comes from the car. We hear the far-off shouting of Nazar. (Farhadi 2014, 62) \{Diegetic sound, Off-screen sound\} 
The wild cries of Nazar shatter the silence of the desert (Farhadi 2014, 64). \{Off-screen sound\}

The car shakes badly, and the wind blows wildly through the broken glass and the plastic cover (Farhadi 2014, 65). \{Ambient sound\}

A silent desert. (Farhadi 2014, 66) \{Ambient sound\}

The sound of a quarrel between the old man and Nazar come from inside the car. (Farhadi 2014, 66) \{Off-screen sound\}

The sound of the wind still comes from the vehicle. (Farhadi 2014, 68) \{Ambient sound\}

A small humble yard. The sound of the doorbell resonates in the yard. (Farhadi 2014, 82) \{Off-screen sound\}

The old man's favourite song is playing on the car's cassette player (Farhadi 2014, 83). \{Diegetic sound, off-screen sound\}

\section{The Beautiful City}

Short synopsis: At a juvenile detention centre, a group of teenagers led by A'la gather to celebrate Akbar's 18th birthday. Akbar is on the death line for murdering a girl two years ago, and now is the time for his execution. A'la and Akbar's sister (Firoozeh) persistently try to gain the plaintiff's consent (Abolghasem, the victim's father). The reluctant father goes to court to speed up the execution, but they tell him he must wait and prepare the blood money for taking Akbar's life. Abolghasem does not afford it, so he tries to finance it from the local mosque, but no one is willing to sign the required testimony.

Meanwhile, Firoozeh - separated from her addicted husband - and A'la fall in love in the course of the film. Abolghasem's wife, who prefers to take her deceased daughter's blood money to spend it on her disabled daughter's treatment, asks Abolghasem to consent. But now, Firoozeh is the one who cannot afford the blood money to pay for his brother's release. After consulting with her brother, Abolghasem's wife lays down one condition to forget about the blood money: A'la, who seems like a capable young man, should marry her disabled daughter. She tells Firoozeh about this condition and asks her to arrange this marriage. A'la, who does not know about the condition yet, tells Firoozeh that he intends to provide the blood money in any way possible, but Firoozeh bitterly opposes the idea. When A'la knows about Abolghasem's family's intentions, he asks Firoozeh to make the final decision. Firoozeh pretends that she does not love A'la. The social workers of the detention centre are also not able to dissuade Abolghasem about his condition. A'la goes back to Firoozeh, but she does not open the door to him.

The noise of the young crowd comes from the yard of the detention centre. (Farhadi 2014, 87) \{Off-screen sound, ambient sound\}

Akbar hears A'la, who came here to see him. (Farhadi 2014, 89) \{Off-screen sound\}

The children's joyous hubbub comes from outside. (Farhadi 2014, 92) \{Off-screen sound, ambient sound\}
Children are playing around the railway, and there is a sound coming from each house. (Farhadi 2014, 99) \{Off-screen sound\}

The dispute between man and woman erupts. The sound of a passing train covers their quarrel. (Farhadi 2014, 102) \{Off-screen sound\}

The sound of the doorbell brings him back to reality. (Farhadi 2014, 103) \{Off-screen sound\}

A noisy train passes by-the doorbell rings. (Farhadi. 2014, 105) \{Off-screen sound\}

A'la is not yet so far when he hears a scream coming from Abolghasem's house. (Farhadi 2014, 110) \{Off-screen sound\}

The voices of Abolghasem and his wife can be heard from outside. (Farhadi 2014, 113) \{Off-screen sound\}

The cries of the baby come from outside the frame. (Farhadi 2014, 118) \{Off-screen sound\}

We hear the door of the room creaks open, and the kiosk owner grunts. (Farhadi 2014, 118) \{Off-screen sound, De-acousmatization\}

The doorbell rings. (Farhadi 2014,

\{Off-screen sound\}

The murmuring of the clients comes from outside. (Farhadi 2014, 119) \{Off-screen sound, ambient sound\} Firoozeh whispers something from outside the frame. (Farhadi 2014, 126) \{Off-screen sound\}

The imam's preaching comes from the loudspeakers. (Farhadi 2014, 128) \{Off-screen sound\}

The sound of people sending Salawat comes from the mosque. (Farhadi 2014, 128) \{Off-screen sound\}

A pleasant song is playing. (Farhadi. 2014, 129) \{Diegetic sound, Off-screen sound\}

A'la and Firoozeh are walking by the railroad, coming ahead through the deep silence of the night. (Farhadi 2014, 131) \{Ambient sound\}

The door is unlatched. We hear the conversation between Abolghasem and some other men. (Farhadi 2014, 133) \{Off-screen sound\}

The sound of Azan comes. (Farhadi 2014, 134) \{Off-screen sound, ambient sound\}

Now Abolghasem's weeping comes from inside the room. (Farhadi 2014, 139) \{Off-screen sound\}

The only sound is the creaking of a cradle. (Farhadi $2014,147)$ \{Off-screen sound\}

The sound of a motorbike comes from the alley. (Farhadi 2014, 149) \{Off-screen sound\}

The doorbell rings. (Farhadi 2014, 151) \{Off-screen sound\}

Children are enjoying their break time in the noisy yard. (Farhadi 2014, 153) \{Off-screen sound, ambient sound\}

Abolghasem's wife is sitting on the porch. She hears Ghafouri's voice coming from inside. (Farhadi 2014, 155) $\{$ Off-screen sound\}

Abolghasem's wife (O.S.): Don't stand at the door! Come here! (Farhadi 2014, 156) \{Off-screen sound, De-acousmatization\}

The doorbell is constantly ringing. (Farhadi 2014, 156) $\{$ Off-screen sound\}

The sound of the last ringing. A'la does not get any answer. A loud train passes. (Farhadi 2014, 157) \{Off-screen sound\} 


\section{Fireworks Wednesday}

Short synopsis: The New Year is coming, and everybody is busy with spring cleaning. A newly engaged young housekeeper (Roohangiz or Roohi) goes to the flat of a middle-class family on Fireworks Wednesday. The tense and sad mood prevailed in the house goes hand in hand with the boisterous Fireworks Wednesday to create a stressful and fragile atmosphere. Mozhde, the housewife, who is wearing all black, looks nervous. She suspects that her husband is having an affair with a neighbour causing their marriage to fall apart. In the end, the woman seems to forgive her husband, and they make reconciliation before the New Year. However, the characters of this story will continue to be lonely and distant.

The only thing that breaks the silence of the winter is the voices of those who talk and laugh. (Farhadi 2014, 162) $\{$ Ambient sound\}

A young man is sitting at a desk answering the never-ending phone calls. (Farhadi 2014, 163) \{Off-screen sound\}

As Roohi enters, we hear the sound of clapping coming from the room. (Farhadi 2014, 165) \{Off-screen sound\}

The phone rings. (Farhadi 2014, 165) \{Off-screen sound\}

A woman's voice comes through the door phone. (Farhadi 2014, 168) \{Off-screen sound\}

The sound of opening the door. (Farhadi 2014, 168) \{Off-screen sound\}

A knock on the door. (Farhadi 2014, 169) \{Off-screen sound\}

Morteza closes his eyes. The sound of the vacuum cleaner disturbs his momentary peace. (Farhadi 2014, 170) $\{$ Off-screen sound\}

Morteza wakes up to the sound of the door. (Farhadi $2014,171)$ \{Off-screen sound\}

The sound of the vacuum cleaner arouses his curiosity, so he peeks into the room. (Farhadi 2014, 171) $\{$ Off-screen sound\}

Mozhde knocks on the door of the caretaker's room. The voice of a few children comes from inside. (Farhadi 2014, 174) \{Off-screen sound\}

A distant sound of a telephone comes from a neighbouring flat. (Farhadi 2014, 180) \{Off-screen sound\}

Mozhde puts his ear to the door. She hears a faint sound. (Farhadi 2014, 180) \{Off-screen sound\}

We hear the woman's voice from the next door, the same voice we heard before, through the door phone. (Farhadi 2014, 182) \{Off-screen sound, De-acousmatization\}

The sound coming from the door phone cuts off. (Farhadi 2014, 183) \{Off-screen sound\}

The doorbell rings. (Farhadi 2014, 185) \{Off-screen sound\}

We hear Mozhde and her mother greet at the entrance. (Farhadi 2014, 185) \{Off-screen sound\}

Mojdeh goes to the bathroom when she hears Simin's main door closes. (Farhadi 2014, 189) \{Off-screen sound\}
The sound of filling the kettle while Simin is softly singing a sweet song. (Farhadi 2014, 192) \{Off-screen sound\}

Roohi (O.S.): Do you have any children? (Farhadi 2014, 192) \{Off-screen sound\}

The doorbell rings. (Farhadi 2014, 193) \{Off-screen sound\}

Simin walks towards the door and turns down the music. (Farhadi 2014, 193) \{Diegetic sound, Off-screen sound\}

The sound of closing the toilet door and a little later Mozhde's doorbell ringing. (Farhadi 2014, 199) \{Off-screen sound\}

Roohi closes her eyes and listens to the different sounds of the city. (Farhadi 2014, 202) \{Off-screen sound\}

The plane's sound is still coming from afar, and then we hear the sound of closing the main door. The sound of the aircraft taking off. (Farhadi 2014, 202) \{Off-screen sound\}

The noise of the children comes from the yard. (Farhadi 2014, 206) \{Off-screen sound\}

The doorbell rings. (Farhadi 2014, 216) \{Off-screen sound\}

The sound of closing the door comes from outside and immediately the sound of a quarrel between Mozhde and Morteza. (Farhadi 2014, 218) \{Off-screen sound\}

The sound of firecrackers and fountains comes from everywhere. (Farhadi 2014, 224) \{Off-screen sound, ambient sound\}

The sound of firecrackers in the park is too high. (Farhadi 2014, 226) \{Off-screen sound, ambient sound\}

The sound of firecrackers and the noise of children come from further away. (Farhadi 2014, 228) \{Off-screen sound, ambient sound\}

Now the noise outside is getting farther and quieter. (Farhadi 2014, 228) \{Off-screen sound, ambient sound\}

We hear the car door opens. (Farhadi 2014, 228) \{Off-screen sound\}

The sounds from outside are clearer. (Farhadi 2014, 228) \{Off-screen sound, ambient sound\}

The sound of someone climbing the car and closing the door. (Farhadi 2014, 228) \{Off-screen sound, acousmetre\}

Morteza watches Simin leave in the mirror, amidst the noise of children playing with fireworks. (Farhadi 2014, 228) \{Off-screen sound, ambient sound\}

We hear the sound of the motorcycle driving away. (Farhadi 2014, 231) \{Off-screen sound\}

The sound of the firecracker is loud and makes Simin jump. (Farhadi 2014, 231) \{Off-screen sound\}

Some car alarms are activated, causing a horrible noise while Simin passes by. (Farhadi 2014, 231) \{Off-screen sound\}

The sound of fireworks is still coming. (Farhadi 2014, 232) \{Off-screen sound, ambient sound\} 


\section{About Eli}

Short synopsis: The film tells the story of a couple of friends who travel to the north to spend their holidays. Ahmad is back in Iran after living many years in Germany, and now his friends are trying to find a proper wife for him. That is why Sepideh invited a kindergarten teacher (Eli) to join them. But after Eli disappears on the beach, the story turns into a tragedy. Everyone starts to blame the others, and when Eli's fiancé arrives, they try to persuade Sepideh to paint a negative image of Eli for him.

The sound of flowing traffic comes from inside a charity box installed on the roadside. (Farhadi 2014, 367) \{Off-screen sound, ambient sound\}

The occasional sound of a car braking. Some coins and banknotes fall into the box. (Farhadi 2014, 367) \{Off-screen sound, ambient sound\}

Someone calls the old woman from inside, and she goes in. (Farhadi 2014, 374) \{Off-screen sound\}

The children are responding all at once, but the sound of the sea almost covers their voices. (Farhadi $2014,377)$ (Off-screen sound, ambient sound\}

Voices of children comes from outside. (Farhadi 2014, 377) $\{$ Off-screen sound\}

The sound of children laughing and joking comes from inside the villa. (Farhadi 2014, 384) \{Off-screen sound, ambient sound\}

The noise of children comes from inside. (Farhadi 2014, 384) \{Off-screen sound\}

Eli is standing in the kitchen and the sound of children laughing and joking comes from afar. (Farhadi 2014, 391) \{Off-screen sound\}

Sepideh (O.S.): Did you find it, Eli, or should I come? (Farhadi 2014, 391) \{Off-screen sound\}

Peyman is smoking. The sound of footsteps. Peyman worriedly passes the smoke to Ahmad. (Farhadi 2014, 391) \{Off-screen sound, acousmetre\}

The men are cheerfully playing volleyball at the back of the villa. (Farhadi 2014, 394) \{Off-screen sound\}

Sepideh (O.S.): We came together, and we will go back together. (Farhadi 2014, 396) \{Off-screen sound\}

We hear the voices of the little girl, Nazi and Amir shouting Eli. (Farhadi 2014, 403). \{Off-screen sound\}

Ahmad (O.S.): Sepideh... Sepideh...! (Farhadi 2014, 405) $\{$ Off-screen sound\}

The sound of Nazi and Sepideh crying comes from the kitchen. (Farhadi 2014, 409) \{Off-screen sound\}

Morvarid, who woke up to the quarrel between Amir and Sepideh, is scared and comes to the kitchen. (Farhadi 2014, 412) \{Off-screen sound\}

The voice of Peyman and his wife fighting in whispers comes from the kitchen. (Farhadi 2014 420) \{Off-screen sound\}

The sound of the crowded square, vendors, passers-by and passengers waiting for a taxi. (Farhadi 2014, 434) \{Off-screen sound, ambient sound\}

The sound of a car comes from outside the villa. Everyone comes to the window. (Farhadi 2014, 441) \{Off-screen sound\}
The sound of Alireza and others opening the door and entering the living room. (Farhadi 2014, 454). \{Off-screen sound\}

In the living room, everyone is relieved to hear Sepideh's voice. (Farhadi 1393, 455) \{Off-screen sound\}

The Man (O.S.): Is it her? (Farhadi 1393, 456) \{Off-screen sound, acousmetre\}

\section{A Separation}

Short Synopsis: Simin is ready to migrate from Iran with her husband, Nader, and their daughter, Termeh. But Nader does not want to leave his father, who has Alzheimer, alone. As a result, Simin files for divorce, but the court rejects her request to have custody of their daughter, so Termeh has to return to her father's house. To take care of his father, Nader hires a housekeeper (Razieh). Razieh, who is pregnant, accepted the job without telling her husband (Hodjat) about it. One day, Nader returns home to find his father on the ground with his hands tied to the bed with a scarf and left alone. When Razieh returns, a fierce fight ensues, the consequences of which not only ruin Nader's life but also change his daughter's image of him. Razieh is taken to the hospital due to a miscarriage. She and her husband sue Nader for killing the fetus. Simin and Nader separate, and now Termeh has to decide to choose to live with one of her parents.

The door is open, and the clients' voices and the corridors' noise come from the next room. (Farhadi 2014, 461) \{Off-screen sound, ambient sound\}

Judge (O.S.): What you are saying is not enough reason to get a divorce. (Farhadi 2014, 461) \{Off-screen sound\}

Razieh (O.S.): Excuse me, madam! (Farhadi 2014, 466) $\{$ Off-screen sound $\}$

The voice of the worker who took the piano downstairs comes from the staircase. (Farhadi 2014, 467) $\{$ Off-screen sound\}

We hear the main door of the house opens. (Farhadi 2014, 473) \{Off-screen sound\}

Termeh (O.S.): I don't know. (Farhadi 2014, 481) \{Off-screen sound\}

Nader comes to the kitchen. He hears the doorbell. (Farhadi 2014, 482) \{Off-screen sound\}

Termeh (O.S.): Continuous. (Farhadi 2014, 484) \{Off-screen sound\}

The doorbell rings. (Farhadi 2014, 485) \{Off-screen sound\}

Nader (O.S.): Give the Persian equivalent of the following words. (Farhadi 2014, 485) \{Off-screen sound\}

The joyous noise of a crowd comes from outside. (Farhadi 2014, 489) \{Off-screen sound\}

We hear the main door of the house opens. (Farhadi 2014, 490) \{Off-screen sound\}

Ms Kalani (O.S.): Yes. Hello. (Farhadi 2014, 492) \{Off-screen sound\}

Termeh (O.S.): No... (Farhadi 2014, 493)

\{Off-screen sound\}

Nader (O.S.): Take his clothes off! (Farhadi 2014, 494) $\{$ Off-screen sound\} 
Termeh is standing by her father, worried. At the same time, we hear Razieh knocking on the door. Nader pushes the door. He hears his father moaning. (Farhadi 2014, 497) \{Off-screen sound\}

Now in this situation, Razieh's constant knocking has driven Nader mad. (Farhadi 2014, 497) \{Off-screen sound\}

The door is half-open, and the ambient noise of the clients comes from the next room. (Farhadi 2014, 506) \{Off-screen sound, ambient sound\}

Simin goes to the phone. Nader's message comes from the answering machine. (Farhadi 2014, 514) \{Off-screen sound, diegetic music\}

Nader (O.S.): Hello... Termeh, dear!... I got caught here. They will keep me here tonight. (Farhadi 2014, 514) $\{$ Off-screen sound\}

Hodjat's shouting comes from the corridor. (Farhadi 2014, 523) \{Off-screen sound\}

We hear a neighbour explaining something to the officer while going upstairs and aggressive objections of Hodjat before leaving. (Farhadi 2014, 533) \{Off-screen sound\}

The sound of a noisy quarrel comes from the school principal's office. (Farhadi 2014, 535) \{Off-screen sound\}

We hear the unlocking sound of the door of the apartment. (Farhadi 2014, 550) \{Off-screen sound\}

The continuous ringing of the doorbell phone. (Farhadi 2014, 554) \{Off-screen sound\}

\section{Conclusion}

Asghar Farhadi uses screenplay and its elements as a tool for narrating his subjective world. The sound has a special place in his works, a position derived from emotional emphases and the creation of narratives characteristic of his style. By examining Michel Chion's theories in Farhadi's works and applying these theories to his films, we can conclude that Farhadi, knowing the role of sound and its elements, uses it precisely in writing his screenplays. The use of sound in his works complements the visualised text and creates a dramatic situation in each scene. Farhadi uses off-screen sound in his works to develop an emphatic atmosphere for the events of the scene. Farhadi uses natural ambient sounds to present a real world based on his style.

Other features of writing sound in Asghar Farhadi's screenplays include making the location understandable for the sound crew so that they can choose the proper technical tools and give the sound designers and mixers a variety of selections to design the most suitable audio space even before the production begins.

\section{Bibliography}

\section{Articles:}

Delaram, Farhad. 2016. "Acousmatic Sound Effects in poetic cinema with a study of Bal (Honey) by Semih Kaplanoğlu." In Avanca Cinema International Conference 2016: 420-427 URL: http://www.avanca.org/EN/publicacao. php

Ahadi, Mohammad Nasser. 2009. "Sounds to be seen, a critique on the book Sound and Music in Documentary Films." . 3. 2020; 2 (7 and 8) :201-212. URL: http://labr. faslnameh.org/article-1-201-fa.html

\section{Books:}

Farhadi, Asghar. 2014. Seven screenplays by Asghar Farhadi. Tehran: Cheshmeh.

Chion, Michel. 2015. Audio-vision: sound on screen. Translated by Mohammad Javad Mozaffarian. Tehran: Qoqnoos

Chion, Michel. 2017. The Voice In Cinema. translated by Fattah Mohammadi. Zanjan: Hezareh Sevom 